\author{
Бакулин И.Г.1, 2, Варламичева А.А.1 \\ 1 ГБУз «Московский клинический научно-практический чентр Департамента здравоохранения г. Москвы» \\ (ГБУз МКНЦ ДЗМ); 111123, г. Москва, шоссе Энтузиастов, 86, Российская Федерация \\ 2 Медицинский институт усовершенствования врачей ФГБОУ ВПО «Московский государственный \\ университет пищевых производств» (МИУВ ФГБОУ ВПО «МГУПП»); 125080, г. Москва, Волоколамское ш., 11 \\ Российская Федерация
}

Настоящее практическое руководство для врачей общей практики, гастроэнтерологов и гепатологов содержит специально отобранные объединенные данные, основанные на международных рекомендациях с учетом результатов крупных клинических исследований. Описаны теоретические положения, касающиеся гепаторенального синдрома (определение, критерии диагностики, классификация), острого повреждения почек и спонтанного бактериального перитонита. Приведен алгоритм диагностики при асците для раннего выявления гепаторенального синдрома, представлены основные лечебно-профилактические мероприятия. С позиции доказательной медицины обсуждены принципы терапии с акцентом на критериях эффективности, в том числе прогностических в зависимости от исходных клинико-лабораторных показателей, и правилах мониторинга.

Ключевые слова: гепаторенальный синдром, цирроз печени, асцит, диуретическая терапия, спонтанный бактериальный перитонит, острое повреждение почек, терлипрессин, рекомендации.

\title{
HEPATORENAL SYNDROME: DIAGNOSTIC AND THERAPEUTIC MANAGEMENT
}

\section{Bakulin I.G.1, 2, Varlamicheva A.A.1}

1 Moscow Clinical Scientific Center, Moscow Health Care Department; 86 Shosse Entuziastov, Moscow, 111123, Russian Federation

2 Medical Institute of Postgraduate Education, Moscow State University of Food Production; 11 Volokolamskoe sh., Moscow, 125080, Russian Federation

The practical guidelines on diagnosis and management of hepatorenal syndrome, which could be useful for general medical practitioners, gastroenterologists, and hepatologists, are considered in this paper. Selected recommendations are based on integrated clinical data, international recommendations and results of clinical trials. Beginning from theoretical grounds of hepatorenal syndrome (its definition, diagnostic criteria, and classification), acute kidney injury, and spontaneous bacterial peritonitis, the paper contains diagnostic algorithms (in particular, in ascites, for early revealing hepatorenal syndrome), and main approaches to therapy and prevention. The efficacy criteria and monitoring rules for the terlipressin-based therapy are listed.

Key words: hepatorenal syndrome, liver cirrhosis, ascites, diuretic therapy, spontaneous bacterial peritonitis, acute kidney injury, terlipressin, recommendations.

\section{ВВЕДЕНИЕ}

Представленные рекомендации сгруппированы по принципу практического руководства для врачей общей практики, гастроэнтерологов, гепатологов и основаны на международных рекомендациях и результатах крупных клинических исследований, суммированных в документах Международного общества по изучению асцита (International Ascitis Club - IAC, 2003, 2007) и Европейской ассоциации по изучению печени (European Association for the Study of the Liver - EASL, 2010) [1, 2].
Каждый пункт рекомендаций базируется на данных рандомизированных клинических исследований и имеет соответствующий уровень доказательности - от I до IV:

Іа - доказательства, полученные в метаанализах рандомизированных исследований;

$\mathrm{lb}$ - доказательства, полученные как минимум в одном рандомизированном исследовании;

IIa - доказательства, полученные как минимум в одном хорошо спланированном контролируемом исследовании без рандомизации; 
Ilb - доказательства, полученные как минимум в одном хорошо спланированном полуэкспериментальном исследовании другого типа;

III - доказательства, полученные в хорошо спланированных неэкспериментальных исследованиях, таких как сравнительные, корреляционные исследования и описания клинических случаев («случай - контроль»);

IV - доказательства, полученные из отчетов экспертных комиссий, на основе мнений или клинического опыта авторитетных специалистов.

Каждой рекомендации присваивается определенная степень (А, В или С). Отметим, что нет прямой связи между степенью рекомендации и уровнем доказательности. Например, отсутствие доказательств высокого уровня не исключает возможности дать рекомендации степень А при условии, что имеется богатый клинический опыт и достигнут консенсус экспертов. При определении степени рекомендаций качество научных доказательств, которые лежат в их основе, является важным фактором, но его необходимо сбалансировать с преимуществами и недостатками, полезностью и предпочтениями, а также со стоимостью:

- степень A - рекомендации основаны на клинических исследованиях надлежащего качества и единообразия, касающегося специфических рекомендаций, включая как минимум одно рандомизированное исследование;

- степень В - рекомендации основаны на адекватно проведенных, но не рандомизированных клинических исследованиях;

- степень C - рекомендации разработаны, несмотря на отсутствие применимых клинических исследований надлежащего качества.

\section{ОПРЕДЕЛЕНИЕ И КЛАССИФИКАЦИЯ ГЕПАТОРЕНАЛЬНОГО СИНДРОМА}

Гепаторенальный синдром (ГРС) - это осложнение, развивающееся у больных с асцитом, ассоциированным с циррозом печени, и носящее характер функциональной почечной недостаточности в отсутствие паренхиматозных заболеваний почек. При ГРС отмечается повышение показателя сыво- роточного креатинина более 133 ммоль/л или его увеличение на 50\% от исходного значения. Обязательными критериями ГРС являются:

- наличие цирроза печени, осложненного асцитом;

- уровень сывороточного креатинина более 133 ммоль/л;

- отсутствие шока (любой этиологии);

- отсутствие гиповолемии, подтвержденной данными измерения центрального венозного давления (ЦВД), и/или снижение креатинина в условиях отмены диуретической терапии на фоне применения альбумина (внутривенно) в дозе 1 г/кг/сут (до 100 г/сут) в течение двух суток;

- отсутствие данных о применении нефротоксичных препаратов;

- отсутствие паренхиматозных заболеваний почек (протеинурия менее 0,5 г/сут, микрогематурия), подтвержденное ультразвуковым исследованием (УЗИ) почек.

Основными триггерами развития ГРС служат бактериальные осложнения, в том числе спонтанный бактериальный перитонит (СБП). Частота диагностирования ГРС при СБП - около 30\% [3].

Одним из наиболее частых осложнений, развивающихся у пациентов с циррозом печени при наличии асцита, является почечная дисфункция - в среднем диагностируется у $20 \%$ госпитализированных пациентов [4]. Причины нарушения почечной функции в большинстве случаев имеют функциональную природу, что, как правило, обусловлено изменением объема циркулирующей крови (ОЦК) на фоне подбора адекватной диуретической терапии. Те же состояния функциональной почечной дисфункции, которые не разрешаются после увеличения ОЦК, присущи истинному ГРС. Дифференциальная диагностика и своевременное подтверждение ГРС имеют важное значение в связи с тем, что после верификации ГРС прогноз изменяется: у таких пациентов значительно увеличивается риск летального исхода [5], что требует немедленного пересмотра лечебных мероприятий.

С 2003 г. ГРС принято делить на два типа. ГРС 1-го типа - это стремительно прогрессирующая

Бакулин Игорь Геннадьевич - д-р мед. наук, профессор, зав. отделом гепатологии ГБУз МКНЦ ДЗМ, зав. кафедрой гастроэнтерологии МИУВ ФГБОУ ВПО «МГУПП». Варламичева Анна Андреевна - мл. науч. сотр. отдела гепатологии ГБУз МКНЦ ДЗМ.

Для корреспонденции: Бакулин Игорь Геннадьевич - 111123, г. Москва, шоссе Энтузиастов, 86, Российская Федерация. Тел.: +7 (925) 51865 38. E-mail: igbakulin@yandex.ru

Запрос программы Hepatology Score (HepS) @ 2014 - hepScore@mail.ru

Bakulin Igor' Gennad'evich - MD, PhD, Professor, Head of the Department of Hepatology, Moscow Clinical Scientific Center; Head of the Department of Gastroenterology, Medical Institute of Postgraduate Education, Moscow State University of Food Production. Varlamicheva Anna Andreevna - Researcher of the Department of Hepatology, Moscow Clinical Scientific Center.

Correspondence to: Bakulin Igor' Gennad'evich - 86 Shosse Entuziastov, Moscow, 111123, Russian Federation. Tel.: +7 (925) 518 65 38. E-mail: igbakulin@yandex.ru

Appeal for Hepatology Score (HepS) @ 2014 submit to: hepScore@mail.ru 
функциональная почечная недостаточность, протекающая по классическим канонам острой почечной недостаточности. В большинстве случаев она совпадает по времени с провоцирующим фактором и развивается одномоментно с нарушением функции печени. Чаще всего триггером выступает тяжелый алкогольный гепатит (ранее - острый алкогольный гепатит) или декомпенсированный цирроз печени (тяжесть по шкале CTP (Child - Turcotte - Pugh) более 9 баллов, по шкале MELD (Model for End-stage Liver Disease) - более 17 баллов) при развитии СБП. C 2010 г. основным критерием диагностики ГРС 1-го типа принято считать повышение уровня сывороточного креатинина более чем на $100 \%$ от исходного и/или его увеличение более чем на 221 ммоль/л за короткое время - менее 2 недель.

Ранее «золотым стандартом» диагностики почечной недостаточности у пациентов с циррозом печени было определение уровня сывороточного креатинина. Однако в настоящее время установлено, что сывороточный креатинин у больных с декомпенсированным циррозом печени и асцитом не отображает истинной скорости клубочковой фильтрации (СКФ) [6]. Очевидно, это происходит из-за нарушения образования креатинина из креатина в ткани печени, в результате чего в биохимическом анализе крови определяется более низкий показатель сывороточного креатинина по отношению к фактической СКФ.

Поскольку показатель креатинина сыворотки не всегда корректно отражает степень почечной недостаточности при циррозе печени, в первую очередь у пациентов с асцитом, использование для диагностики ГРС только одного этого показателя может привести к недооценке потенциально значимых клинических состояний почечной дисфункции, особенно когда показатель креатинина не достигает порогового значения для диагностики ГРС.

Нефрологами определено понятие острого повреждения почек (англ. acute kidney injury, AKI) как относительный прирост к абсолютному повышению креатинина в сыворотке крови у пациентов с заболеванием почек [7]. На Международном конгрессе по изучению печени (International Liver Congress), прошедшем в Лондоне в 2014 г., рекомендовано пользоваться этим же подходом в диагностике AKI у пациентов с циррозом печени. Наименьшим повышением уровня сывороточного креатинина, которое способно отразить значительное снижение выживаемости, принято считать 26,4 ммоль/л в течение 48 часов, что соответствует картине ГРС 1-го типа.

ГРС 2-го типа - функциональная почечная недостаточность, прогрессирующая медленнее, чем ГРС 1-го типа, и, как правило, развивающаяся на фоне резистентного асцита. К основным характеристикам ГРС 2-го типа относится устойчивая, но умеренная степень функциональной почечной недостаточности, часто протекающая с задержкой натрия. Пациенты со 2-м типом ГРС относятся к группе риска по развитию ГРС 1-го типа в отличие от пациентов с нормальной функцией почек. Триггерами перехода в ГРС 1-го типа могут выступать бактериальные осложнения, в том числе СБП [8]. ГРС 2-го типа действительно прогрессирует более медленными темпами, но это не исключает возможности развития и/или декомпенсации патологии почек в условиях длительного снижения их перфузии. Основой для развития хронического повреждения почек служит ишемия. Но даже вне зависимости от степени перфузии и ишемии у пациентов с циррозом печени могут быть сопутствующие заболевания, вносящие существенный вклад в хроническое повреждение почек.

Выделяют следующие основные причины острого и хронического повреждения почек у больных с циррозом печени.

Острое повреждение почек:

- ГРС 1-го типа;

- преренальная (функциональная) почечная недостаточность;

- острый тубулярный некроз;

- состояние после ГРС;

- состояние после преренальной (функциональной) почечной недостаточности;

- лекарственно-индуцированное повреждение;

- осмотические тубулопатии.

Хроническое повреждение почек:

- диабетический гломерулосклероз;

- ишемическая нефропатия;

- алкогольная иммуноглобулин А (IgA)ассоциированная нефропатия;

- вирус гепатита C (HCV)-индуцированный мембранопролиферативный гломерулонефрит;

- недиабетический гломерулосклероз;

- ГРС 2-го типа.

\section{АЛГОРИТМ ДИАГНОСТИКИ}

Высокий риск летального исхода при развитии ГРС требует настороженности по отношению ко всем пациентам с асцитом, особенно при наличии признаков СБП. Ниже представлены мероприятия по оценке ряда показателей, отклонения от нормы которых следует рассматривать как фактор риска развития ГРС.

1. Определение уровня сывороточного креатинина

Всем пациентам с асцитом необходимо проводить мониторинг лабораторных показателей 
для выявления одного из патогномоничных признаков ГРС - увеличения показателя креатинина сыворотки:

- повышения в сыворотке более 133 ммоль/л;

- увеличения на 26,4 ммоль/л за 48 часов;

- увеличения на $50 \%$ от исходного уровня (при увеличении более $100 \%$ от исходного диагностируется ГРС 1-го типа).

2. Определение натрия в сыворотке

3. Дифференциальная диагностика преренальной азотемии и острого повреждения почек

В таблице отображена дифференциальная диагностика поражений почек методом определения фракционной экскреции натрия (Fractional excretion of Sodium - $\mathrm{FE}_{\mathrm{Na}}$ ).

Вариант поражения почек в зависимости от фракционной экскреции натрия

\begin{tabular}{l|c|c|c}
\hline \multirow{2}{*}{ Показатели } & \multicolumn{3}{|c}{ Фракционная экскреция натрия } \\
\cline { 2 - 4 } & преренальная & ренальная & постренальная \\
\hline $\mathrm{FE}_{\mathrm{Na}}$ & $<1 \%$ & $>1 \%$ & $>4 \%$ \\
$\begin{array}{l}\text { Натрий } \\
\text { в моче, } \\
\text { ммоль/л }\end{array}$ & $<20$ & $>40$ & $>40$
\end{tabular}

4. Измерение диуреза

5. Определение суточной экскреции натрия с мочой, исключение паренхиматозных заболеваний почек (В1):

- клинический анализ мочи (исключение протеинурии, гематурии);

- узИ почек;

- определение экскреции калия с мочой (дифференциальный диагноз заболеваний почек);

- биопсия почек (проводится при невозможности исключения паренхиматозного заболевания почек, рекомендовано для пациентов - кандидатов на сочетанную трансплантацию печени и почки).

6. Определение калия в сыворотке (для оценки развития диуретикассоциированных осложнений)

7. Исключение гиповолемии (измерение ЦВД)

8. Исключение септического шока

9. Скрининг бактериальных инфекций во всех средах (моча, кровь, асцитическая жидкость)

10. Диагностика СБП

В связи с тем, что ГРС в $30 \%$ случаев развивается на фоне СБП, одним из основных методов дополнительной диагностики является определение и профилактика провоцирующих факторов и предрасполагающих состояний. Для исключения СБП необходимо помнить об основных диагностических мероприятиях, осуществляемых у пациентов с асцитом на фоне цирроза печени. К таким мероприятиям относятся диагностический парацентез, анализ асцитической жидкости, сопоставление лабораторных результатов диагностики асцитической жидкости и сыворотки. При проведении диагностического парацентеза должна быть выполнена диагностика СБП. Диагностический парацентез необходимо проводить всем больным с подозрением на развитие ГРС (A1).

СБП устанавливается при наличии любого из следующих условий (A1):

- абсолютное число нейтрофильных лейкоцитов более $250 / \mathrm{mm}^{3}$;

- определение положительного роста культуры при посеве асцитической жидкости (даже при количестве лейкоцитов менее $250 / \mathrm{Mm}^{3}$ );

- снижение уровня белка в асцитической жидкости менее 15 г/л.

Подтверждение диагноза ГРС возможно при наличии у пациента всех основных критериев:

- цирроза печени;

- асцита;

- креатинина сыворотки, превышающего 133 ммоль/л, или его увеличение на 50\% от исходного значения (если отмечается повышение уровня креатинина на $100 \%$ от исходного значения, диагностируется ГРС 1-го типа);

- нормального размера почек по данным УзИ;

- отсутствия патологических отклонений в клиническом анализе мочи;

- $\mathrm{FE}_{\mathrm{Na}}<1 \%$;

- ЦВД от 50 до 120 мм вод. ст.

Кроме того, возможно наличие дополнительных критериев, указывающих на развитие ГРС:

- олигурия (диурез менее 400 мл/сут) или анурия (диурез менее 200 мл/сут);

- натрий сыворотки менее 130 ммоль/л;

- экскреция натрия менее 30 ммоль/л;

- СБП и/или другие бактериальные инфекции.

\section{ЛЕЧЕНИЕ ГЕПАТОРЕНАЛЬНОГО СИНДРОМА}

Из-за прогрессирования почечной недостаточности при подозрении/верификации ГРС лечение должно быть назначено незамедлительно. Не следует чрезмерно вводить жидкость, избегая возможного развития гиперволемии и/или гипонатриемии разведения. Применение калийсберегающих диуретиков при развитии ГРС следует отменить вследствие повышения риска развития тяжелой гиперкалиемии (A1).

\section{Препараты, которые необходимо отменить} При подозрении на ГРС следует отменить все диуретические препараты пациентов. В среднем дифференциальная диагностика ГРС с гиповолемией 
и почечной дисфункцией другой этиологии занимает 48 часов. В течение этого периода спиронолактон, фуросемид и другие диуретические препараты следует отменить (A1).

У всех пациентов с диагностированным ГРС все диуретические препараты должны быть отменены (A1).

У пациентов с текущим ГРС 1-го типа нет данных об использовании фуросемида. Тем не менее фуросемид может быть применен для стимулирования диуреза и снижения гиперволемической перегрузки (только для эпизодического использования и при условии показателя натрия сыворотки больше 120 ммоль/л) (А1). Следует помнить, что фуросемид относится к нефротоксичным препаратам. Спиронолактон противопоказан из-за высокого риска развития жизнеугрожающей гиперкалиемии (A1).

Следует отменить такие нефротоксичные препараты, как нестероидные противовоспалительные средства (ибупрофен, ацетилсалициловая кислота, диклофенак), рентгеноконтрастные вещества для внутривенного введения, аминогликозиды, амфотерицин В, сульфаниламиды, иммуносупрессанты, цисплатин, иммуноглобулины, маннитол, противоопухолевые, литий- и фторсодержащие препараты, циклофосфамид, метотрексат, ацикловир, этиленгликоль, ранитидин, циметидин, фенитоин, пеницилламин, демеклоциклин, фоскарнет натрий, фуросемид.

\section{Препараты, которые необходимо назначить}

\section{1. Вазоконстрикторы}

В настоящее время наибольшее значение в лечении ГРС отводится применению аналогов вазопрессина. В многочисленных клинических исследованиях получена достоверная доказательная база применения терлипрессина, зарекомендовавшего себя в клиническом применении как препарат выбора [9, 10, 11, 12, 13, 14, 15, 16, 17, 18]. В среднем эффективность применения терлипрессина при ГРС 1-го типа составляет 40-50\% [9, 19]. Использование вазоконстрикторов изучено только в комбинации с внутривенным введением альбумина, то есть следует рассматривать совместное применение данных препаратов.

В отсутствие положительной динамики в виде снижения креатинина сыворотки на 25\% в течение 3 дней следует повысить рекомендуемую начальную дозу терлипрессина- 1 мг каждые 4-6 часов- до 2 мг стакой же периодичностью приема. Средняя продолжительность применения терлипрессина составляет 14 дней, длительность терапии напрямую зависит от исходного повышения уровня креатинина. Превышать длительность двухнедельного курса терапии не рекомендуется.
Для пациентов с ранним рецидивом ГРС 1-го типа терлипрессин является препаратом выбора с доказанной эффективностью. Важнейшим обоснованием применения терлипрессина служат достоверные данные повышения выживаемости. Так, результаты метаанализа, включающего восемь клинических исследований с совокупным участием 377 больных с ГРС, свидетельствуют о том, что применение терлипрессина способствовало снижению летальности в ближайший (госпитальный) и отдаленный период на 9-15\%. Кроме того, было отмечено повышение выживаемости больных с ГРС при комбинированном применении низких доз терлипрессина (менее 4 мг/сут) и альбумина [20].

2. Альтернативные схемы применения вазоконстрикторов:

- октреотид (100-200 мкг 3 раза в сутки подкожно) + мидодрин (2,5-12,5 мг);

- октреотид (100-200 мкг 3 раза в сутки подкожно) + норэпинефрин (0,5-3 мг/ч внутривенно).

Указанные схемы возможны к применению только в комбинации с альбумином в адекватных дозах. В первые 3 дня лечения альбумин в комбинации с вазоконстрикторами вводится в дозе 1 г/кг/сут, далее - 40 г/сут. В случае диагностирования СБП доза альбумина в первые 3 дня лечения составляет 1,5 г/кг/сут, далее 1 г/кг/сут до разрешения СБП; максимальная суточная доза альбумина - 100 г.

\section{3. Антибактериальная терапия}

При наличии признаков бактериальной инфекции (кровь, моча) следует незамедлительно назначить антибиотики широкого спектра действия. У пациентов, получающих профилактический курс антибактериальных препаратов, терапия должна быть продолжена. Назначение антибактериальных препаратов широкого спектра действия эмпирически, без установления источника инфекции, при ГРС 1-го типа в настоящее время не рекомендовано (С1).

Профилактически антибиотики должны быть назначены всем пациентам с асцитом и подозрением на ГРС в отсутствие признаков СБП. В этом случае, как и у пациентов с купированными проявлениями СБП, препаратом выбора служит норфлоксацин (400 мг/сут). Альтернативными препаратами являются ципрофлоксацин (750 мг 1 раз в сутки) или ко-тримоксазол (сульфаметоксазол 800 мг/сут + триметоприм 160 мг/сут). При низком содержании белка в асцитической жидкости (менее 15 г/л) данное состояние расценивается как фактор риска развития СБП, что требует профилактического назначения норфлоксацина в дозе 400 мг/сут.

При диагностировании СБП незамедлительно назначается эмпирическая антибиотикотерапия. 
Препаратами выбора являются цефалоспорины 3-4-го поколения в дозе 4 г/сут с обязательным проведением повторного диагностического парацентеза через 48 часов и на 5-е сутки применения антибиотиков для контроля эффективности антибактериальной терапии. Антибиотикотерапия при СБП проводится только в комбинации с альбумином (внутривенно).

\section{4. Бета-адреноблокаторы}

Относительно препаратов группы $\beta$-адреноблокаторов, которые рекомендованы для постоянного применения у пациентов с варикозно-расширенными венами пищевода 2-4-й степеней, нет достоверных данных о необходимости отмены или продолжения терапии (B1).

\section{5. Парацентез}

Нет данных, свидетельствующих против проведения парацентеза с адекватным возмещением альбумина у пациентов с асцитом 3-й степени при развитии ГРС 1-го типа. Данный метод может быть основным при симптоматической терапии для разрешения дискомфорта пациента (B1).

\section{Альтернативные методы лечения}

Применение альтернативных методов не имеет доказанной большей эффективности, так как прицельные клинические исследования их сравнения с применением вазоконстрикторов не проводились, но они всегда могут быть рассмотрены у пациентов с непереносимостью и/или наличием противопоказаний к применению терлипрессина. К альтернативным методам относятся:

- трансъюгулярное внутрипеченочное портосистемное шунтирование (англ. transjugular intrahepatic portosystemic shunt - TIPS);

- гемодиализ;

- гемофильтрация;

- альбуминовый диализ при помощи молекулярной адсорбирующей рециркулирующей системы (англ. molecular absorbent recirculating system-MARS);

- система экстракорпоральной поддержки функции печени Prometeus;

- трансплантация печени при лечении ГРС 1-го типа - метод выбора, который повышает выживаемость до 65\%. Все пациенты с этим заболеванием имеют приоритет в листе ожидания из-за высокого риска летального исхода;

- трансплантация печени рекомендована также как метод выбора при лечении ГРС 2-го типа.

\section{Критерии эффективности лечения гепаторенального синдрома}

Критериями эффективности служат:

- достижение целевого значения креатинина ниже 133 ммоль/л на фоне проводимой терапии комбинацией терлипрессина и альбумина в течение 14 дней;

- изменения клинико-лабораторных показателей: снижение креатинина сыворотки, повышение артериального давления, увеличение объема выделяемой мочи, повышение показателя натрия сыворотки.

\section{Правила мониторирования}

Пациенты с установленным ГРС должны подвергаться тщательному мониторингу для оценки таких клинико-лабораторных данных, как подсчет диуреза, определение баланса жидкости (объем выпитой + внутривенной жидкости / объем диуреза), показатели гемодинамики (частота сердечных сокращений, артериальное давление), температура тела, клинический анализ крови, биохимический анализ крови (креатинин, мочевина, натрий, калий, билирубин общий, аланинаминотрансфераза, альбумин), клинический анализ мочи, суточная экскреция натрия и калия.

Идеальным считается наблюдение пациента с ГРС в палате или отделении интенсивной терапии. В таких условиях возможность мониторирования ЦВД поможет избежать гиперволемии (A1).

\section{ПРОГНО3}

Важность диагностики ГРС и его деления на типы обусловлена в первую очередь тем, что нарушение почечной функции у больных с асцитом сопровождается высоким риском летального исхода. В отсутствие противопоказаний эти пациенты рассматриваются как первоочередные кандидаты для трансплантации печени.

Риск летального исхода при развитии ГРС 1-го типа составляет 80\% в течение 90 дней; как правило, медиана летальности приходится на госпитальный период - до 30 дней [21, 22]. Для расчета риска летального исхода при ГРС 2-го типа принято руководствоваться соответствующими значениями шкалы MELD, что укладывается в диапазон от 1,9 до 71,3\% смертности [23]. Ниже представлен прогноз летальности в зависимости от индекса MELD в баллах:

Более 40 баллов . . . . 71,3\% трехмесячной летальности

30-39 баллов. . . . . . . . 52,6\% - "-

20-29 баллов. . . . . . . . . 19,6\% - "-

10-19 баллов. . . . . . . . . 6,0\% - "-

Менее 9 баллов . . . . . 1,9\% - "-

Для упрощения диагностики жизнеугрожающих состояний у больных циррозом печени сотрудниками научно-исследовательского отдела гепатологии Московского клинического научнопрактического центра Департамента здравоохра- 
нения г. Москвы разработана программа для персонального компьютера Hepatology Score(C2014 (HepS(C), которая является доступным инструментом «рабочего стола» врача амбулаторной и стационарной практики ${ }^{1}$. Уникальность программы HepS(C) заключается в том, что это первый русскоязычный проект расчета диагностических и прогностических индексов (MELD, CTP, FENa, Lille Model и др.), а также терапевтических алгоритмов у больных с различными заболеваниями печени с позиции доказательной медицины. Доступ к пилотной версии программы HерS( ) реализуется через запрос по электронной почте HepScore@mail.ru.

\section{Предикторы неблагоприятного исхода при гепаторенальном синдроме}

Рядом авторов выделены предикторы раннего летального исхода (до 30 дней):

- повышение общего билирубина сыворотки более 170 мкмоль/л расценивается как дополнительное эндогенное нефротоксичное действие;

- исходно высокие значения сывороточного креатинина (более 450 ммоль/л) рассматриваются как признак тяжелой труднокорригируемой почечной дисфункции и продолжающегося ГРС.

Такие пациенты имеют крайне неблагоприятный прогноз.

Неблагоприятные предикторы ответа на терапию терлипрессином:

- развитие ГРС по причине алкогольного гепатита $(p<0,001)$;

- высокий уровень креатинина сыворотки на момент начала терапии ( $p<0,001)$;

- высокие значения индексов MELD $(p=0,03)$, CTP $(p=0,05)[24]$.

\section{ПРОФИЛАКТИКА}

В отсутствие признаков СБП (при условии подтверждения данными исследования асцитической жидкости) всем пациентам с асцитом показано профилактическое назначение антибиотиков. Препаратом выбора является норфлоксацин (400 мг/сут), альтернативные препараты - ципрофлоксацин 750 мг 1 раз в неделю или ко-тримоксазол (сульфаметоксазол 800 мг/сут в комбинации с триметопримом 160 мг/сут). При низком содержании белка в асцитической жидкости (менее 15 г/л) показано применение норфлоксацина в дозе 400 мг/сут, поскольку данное состояние расценивается как фактор развития СБП.

Пациентам с диагностированным СБП незамедлительно назначается эмпирическая антибиотикотерапия: препаратами выбора являются цефалоспорины начиная с 3-го поколения в дозе 4 г/сут с обязательным проведением повторного диагностического парацентеза для контроля эффективности антибиотикотерапии через 48 часов и на 5-е сутки приема антибиотиков. Лечение СБП проводится только комбинацией антибиотиков с альбумином (A1). После разрешения СБП назначается профилактическая антибиотикотерапия (A1).

Применение в качестве профилактики пентоксифиллина (400 мг 3 раза в день в течение 4 недель) у пациентов с алкогольным гепатитом, по мнению ряда авторов [25], предотвращает развитие ГРС (B2), но не имеет доказательной базы при развитии ГРС у больных циррозом печени.

Учитывая низкую эффективность терапевтических мероприятий и плохой прогноз при развитии ГРС, все пациенты с асцитом 2-3-й степени рассматриваются как кандидаты на проведение трансъюгулярного внутрипеченочного шунтирования (TIPS) для хирургического симптоматического лечения и на донорскую или ортотопическую трансплантацию печени как радикального метода лечения. Следует подчеркнуть, что все пациенты с впервые выявленным асцитом должны быть поставлены в лист ожидания трансплантации печени (A1).

\section{ЗАКЛЮЧЕНИЕ}

Несмотря на успехи медицины ГРС остается смертельным осложнением асцита, ассоциированного с циррозом печени, который имеет высокую частоту летальности, в том числе госпитальной (в пятнадцати-тридцатидневный срок). В течение последних нескольких лет практические рекомендации для врачей по ведению больных с ГРС претерпели многочисленные изменения, и данные, приведенные в настоящей статье, суммируют современные диагностические подходы, принципы назначения лечебно-профилактических мероприятий и критерии их эффективности.

К сожалению, приходится констатировать, что в настоящее время в различных лечебных учреждениях Российской Федерации существуют разные подходы к диагностике и лечению больных с ГРС или при подозрении на него. В этой связи, на наш взгляд, требуются национальные рекомендации, в которых с позиции доказательной медицины будут представлены диагностические мероприятия и лечебно-профилактические алгоритмы по ведению пациентов с ГРС. Учитывая особенности организации системы здравоохранения в нашей стране, врачам, курирующим больных с декомпенсированными заболеваниями печени, с момента выявле-

1 Hepatology Score - Chronic Hepatitis C (НерSС). Свидетельство на программу эBM №2014617213 от 16.07.2014. Автор - А.А. Варламичева. 
ния первого эпизода асцита рекомендуется консультация трансплантолога с целью постановки пациента в лист ожидания трансплантации печени, так как развитие ГРС и других осложнений асцита сопряжено с высоким риском смерти.

\section{Литература}

1. European Association for the Study of the Liver. EASL clinical practice guidelines on the management of ascites, spontaneous bacterial peritonitis, and hepatorenal syndrome in cirrhosis. J Hepatol. 2010;53(3):397-417.

2. Runyon BA; Practice Guidelines Committee, American Association for the Study of Liver Diseases (AASLD). Management of adult patients with ascites due to cirrhosis. Hepatology. 2004;39(3):841-56

3. Sort $\mathrm{P}$, Navasa M, Arroyo V, Aldeguer X, Planas R, Ruiz-del-Arbol L, Castells L, Vargas V, Soriano G, Guevara M, Ginès P, Rodés J. Effect of intravenous albumin on renal impairment and mortality in patients with cirrhosis and spontaneous bacterial peritonitis. N Engl J Med. 1999;341(6):403-9.

4. Garcia-Tsao G, Parikh CR, Viola A. Acute kidney injury in cirrhosis. Hepatology. 2008;48(6):2064-77.

5. Salerno F, Gerbes A, Ginès P, Wong F, Arroyo V. Diagnosis, prevention and treatment of hepatorenal syndrome in cirrhosis. Gut. 2007;56(9):1310-8.

6. Caregaro L, Menon F, Angeli P, Amodio P, Merkel C, Bortoluzzi A, Alberino F, Gatta A. Limitations of serum creatinine level and creatinine clearance as filtration markers in cirrhosis. Arch Intern Med. 1994;154(2):201-5.

7. Mehta RL, Kellum JA, Shah SV, Molitoris BA, Ronco C, Warnock DG, Levin A; Acute Kidney Injury Network. Acute Kidney Injury Network: report of an initiative to improve outcomes in acute kidney injury. Crit Care. 2007;11(2):R31.

8. Arroyo V, Ginès P, Gerbes AL, Dudley FJ, Gentilini P, Laffi G, Reynolds TB, Ring-Larsen $\mathrm{H}$, Schölmerich J. Definition and diagnostic criteria of refractory ascites and hepatorenal syndrome in cirrhosis. International Ascites Club. Hepatology. 1996;23(1):164-76.

9. Ginès $P$, Schrier RW. Renal failure in cirrhosis. N Engl J Med. 2009;361(13):1279-90.

10. Kew MC, Varma RR, Sampson DJ, Sherlock S. The effect of octapressin on renal and intrarenal blood flow in cirrhosis of the liver. Gut. 1972;13(4):293-6.

11. Lenz K, Hörtnagl H, Druml W, Reither H, Schmid R, Schneeweiss B, Laggner A, Grimm G, Gerbes AL. Ornipressin in the treatment of functional renal failure in decompensated liver cirrhosis. Effects on renal hemodynamics and atrial natriuretic factor. Gastroenterology. 1991;101(4):1060-7.

12. Guevara $M$, Ginès $P$, Fernández-Esparrach $G$, Sort $P$, Salmerón JM, Jiménez $\mathrm{W}$, Arroyo $\mathrm{V}$, Rodés J. Reversibility of hepatorenal syndrome by prolonged administration of ornipressin and plasma volume expansion. Hepatology. 1998;27(1):35-41.

13. Gülberg V, Bilzer M, Gerbes AL. Long-term therapy and retreatment of hepatorenal syndrome type 1 with ornipressin and dopamine. Hepatology. 1999;30(4):870-5.

\section{Конфликт интересов}

Авторы сообщают об отсутствии конфликта интересов и финансовой заинтересованности в ходе написания данной статьи и не несут ответственности за наличие и содержание рекламных материалов, содержащихся на страницах журнала.

14. Moreau R, Durand F, Poynard T, Duhamel C, Cervoni JP, Ichaï $P$, Abergel A, Halimi C, Pauwels $M$, Bronowicki JP, Giostra E, Fleurot $C$, Gurnot $D$, Nouel $O$, Renard $P$, Rivoal $M$, Blanc $P$, Coumaros D, Ducloux S, Levy S, Pariente A, Perarnau JM, Roche J, Scribe-Outtas M, Valla D, Bernard B, Samuel D, Butel J, Hadengue A, Platek A, Lebrec D, Cadranel JF. Terlipressin in patients with cirrhosis and type 1 hepatorenal syndrome: a retrospective multicenter study. Gastroenterology. 2002;122(4):923-30.

15. Fabrizi F, Dixit V, Martin P. Meta-analysis: terlipressin therapy for the hepatorenal syndrome. Aliment Pharmacol Ther 2006;24(6):935-44.

16. Gluud LL, Kjaer MS, Christensen E. Terlipressin for hepatorenal syndrome. Cochrane Database Syst Rev. 2006;(4):CD005162.

17. Sanyal AJ, Boyer T, Garcia-Tsao G, Regenstein F, Rossaro L, Appenrodt B, Blei A, Gülberg V, Sigal S, Teuber P; Terlipressin Study Group. A randomized, prospective, double-blind, placebo-controlled trial of terlipressin for type 1 hepatorenal syndrome. Gastroenterology. 2008;134(5):1360-8.

18. Martín-Llahí $M$, Pépin MN, Guevara M, Díaz F, Torre A, Monescillo $A$, Soriano $G$, Terra $C$, Fábrega $E$, Arroyo $V$, Rodés J, Ginès $P$; TAHRS Investigators. Terlipressin and albumin vs albumin in patients with cirrhosis and hepatorenal syndrome: a randomized study. Gastroenterology. 2008;134(5):1352-9.

19. Moreau $R$, Lebrec $D$. The use of vasoconstrictors in patients with cirrhosis: type 1 HRS and beyond. Hepatology. 2006;43(3):385-94.

20. Hiremath SB, Srinivas LD. Survival benefits of terlipressin and non-responder state in hepatorenal syndrome: a meta-analysis. Indian J Pharmacol. 2013;45(1):54-60.

21. Gines P, Arroyo V, Rodes J, Schrier R, editors. Ascites and renal dysfunction in liver disease. $2^{\text {nd }}$ ed. Oxford: Blackwell Publishing; 2005.

22. Alessandria $C$, Ozdogan $O$, Guevara $M$, Restuccia $T$, Jiménez $W$, Arroyo $\mathrm{V}$, Rodés J, Ginès P. MELD score and clinical type predict prognosis in hepatorenal syndrome: relevance to liver transplantation. Hepatology. 2005;41(6):1282-9.

23. Wiesner R, Edwards E, Freeman R, Harper A, Kim R, Kamath $P$, Kremers W, Lake J, Howard T, Merion RM, Wolfe RA, Krom R; United Network for Organ Sharing Liver Disease Severity Score Committee. Model for end-stage liver disease (MELD) and allocation of donor livers. Gastroenterology. 2003;124(1):91-6.

24. Boyer TD, Sanyal AJ, Garcia-Tsao G, Blei A, Carl D, Bexon AS, Teuber $P$; Terlipressin Study Group. Predictors of response to terlipressin plus albumin in hepatorenal syndrome (HRS) type 1: relationship of serum creatinine to hemodynamics. J Hepatol. 2011;55(2): 315-21.

25. Akriviadis E, Botla R, Briggs W, Han S, Reynolds T, Shakil O. Pentoxifylline improves short-term survival in severe acute alcoholic hepatitis: a double-blind, placebo-controlled trial. Gastroenterology. 2000;119(6):1637-48. 